\title{
ALL-ATMOSPHERE IS-MST RADAR
}

\author{
A.V. Medvedev \\ Institute of Solar-Terrestrial Physics SB RAS, \\ Irkutsk, Russia, medvedev@iszf.irk.ru \\ A.P. Potekhin $\dagger$ \\ Institute of Solar-Terrestrial Physics SB RAS, \\ Irkutsk, Russia
}

\section{A.G. Setov}

Institute of Solar-Terrestrial Physics SB RAS,

Irkutsk,Russia,setov@iszf.irk.ru

\author{
D.S. Kushnarev \\ Institute of Solar-Terrestrial Physics SB RAS, \\ Irkutsk,Russia,ds_k@iszf.irk.ru
}

V.P. Lebedev

Institute of Solar-Terrestrial Physics SB RAS,

Irkutsk,Russia,lebedev@isz.irk.ru

\begin{abstract}
The IS-MST radar, as the name implies, combines two different methods for studying the atmosphere with a backscatter signal. Turbulent fluctuations of the medium cause scattering in the mesospherestratosphere-troposphere (MST). In the upper atmosphere, incoherent scatter (IS) occurs in ionospheric plasma. Special-purpose instruments have been built before such that measurements in one of these modes were the most effective. MST radars were utilized for studying wave activity in the lower and middle atmosphere; the IS radar, for ionospheric research. Nowadays, however, for a comprehensive investigation of atmospheric phenomena, it is necessary to understand processes in all atmospheric layers and near-Earth space. The radar, which combines capabilities of IS and MST measurements, will be able to cover layers from the troposphere to the plasmasphere and to study processes of energy transfer from the lower and middle atmosphere to the ionosphere as well as the interaction of the magnetosphere with the upper atmosphere. Apart from
\end{abstract}

atmospheric research, the radar will allow us to track spacecraft and space debris, determining precise coordinate characteristics. The antenna system is also suitable for radio astronomical observations. In the paper, we provide justification for the 154-162 MHz frequency range and discuss technical solutions of the IS-MST radar project and basic operating modes. In addition, we estimate radar diagnostic capabilities for measurements of different types.

Keywords: IS-MST radar, National Heliogeophysical Complex, incoherent scatter, mesosphere-stratospheretroposphere radar, ionosphere, atmosphere.

\section{INTRODUCTION}

The atmosphere and near-Earth space are complex interconnected systems whose study requires widerange instruments. It is necessary to study not only individual layers of the systems - the lower and middle neutral atmosphere, the ionosphere, plasmasphere, and magnetosphere - but also their interaction.

Ionosphere conditions have a significant effect on human activity. Increased solar activity causes geomagnetic storms, which generate ionospheric disturbances and irregularities. The disturbances and irregularities change radio wave propagation characteristics, thereby causing signals between ground stations and satellites to fade and the direction of propagation along shortwave radio paths to change. Moreover, electric fields generated in the ionosphere induce currents in long conducting lines (electric lines and pipelines), which leads to premature deterioration of equipment. Powerful storms may cause disruption or destruction of the conducting lines. Dynamics of the upper atmosphere is largely determined by magnetospheric processes. During storms, energetic particles precipitate from the radiation belt, and powerful electric fields and currents appear in the magnetosphere. This, in turn, leads to a change in ionization and generates currents in the ionosphere. For space weather monitoring it is necessary to carefully and regularly monitor the state of the ionosphere and to examine the interaction between the magnetosphere and the upper atmosphere.

While the magnetosphere affects the upper atmosphere from above, the dynamics and chemical processes in the troposphere, stratosphere, and mesosphere (MST region) affect it from bellow. The MST region determines the structure and ion composition of ionospheric plasma and influences the redistribution of energy between different regions of the upper and middle atmosphere. Planetary waves (PWs) and mesoscale waves, among which internal gravity waves (IGWs) play the main role, appear in the troposphere and propagate to the stratosphere, mesosphere, and thermosphere, leading to strong vertical interactions. Waves of all scales are generated by meteorological processes, earthquakes, explosions, etc. The waves affect the overall atmosphere dynamics and transfer energy and momentum to the upper atmosphere.

The fundamental problem of modern solar-terrestrial physics is a quantitative description of these processes, assessment of capabilities of deterministic prediction of interactions in the Sun-Earth system, development of modern effective models for forecasting the state of the atmosphere and near-Earth space, which take into account the entire range of horizontal and vertical relations in the system. 
The National Heliogeophysical Complex of the Russian Academy of Sciences (NHC RAS) includes a whole cluster of radio physical instruments for studying space weather, processes in the ionosphere and atmosphere. The most informative instrument of the cluster is the IS-MST radar, which provides vertical profiles of different physical parameters in a great height range 10$1500 \mathrm{~km}$. It covers both the mesospherestratosphere - troposphere (MCT) region, where data is obtained by scattering of radio waves by turbulence and irregularities in the refractive index of the neutral atmosphere, and the ionosphere and plasmasphere, where measurements are made using the incoherent scatter (IS) method. The IS-MST radar is being developed by OAO RTI (Moscow) on the basis of modern digital equipment with antenna system as phased array.

The IS method provides detailed information about the ionosphere at individual heights in a localized region and is not bounded by the heights below the ionization maximum. Modern IS radars measure plasma parameters in several independent vertical slices of the ionosphere, making it possible to determine the three-dimensional structure of irregularities, characteristics of disturbances, and their direction. This method relies on wave scattering by thermal fluctuations of electrons. Since electrons and ions in ionospheric plasma interact, the backscatter signal has a complex spectrum whose components correspond to different plasma oscillations. The spectrum shape depends on physical parameters of the ionosphere, which can be determined by solving the inverse problem for IS. Using the ionacoustic spectrum component, we can restore the electron density, electron and ion temperatures, plasma drift velocity in the radial direction, ionic mass, and electric field strength [Evans, 1969]. The electron line of the IS spectrum has a frequency shift equal to plasma frequency, and the amplitude of the line depends on electron density. Since ionospheric plasma is in the magnetic field, the Faraday effect occurs - rotation of the propagating wave polarization plane, with the rate of rotation also dependent on electron density. The IS-MST radar will have all necessary characteristics to measure parameters of the ionosphere from the $\mathrm{D}$ and $\mathrm{E}$ layers to the plasmasphere due to the small radio horizon and flexibility in the choice of operating modes.

In addition to wave activity, in the lower and middle atmosphere high turbulences and horizontally extended layers appear which lead to partial reflection of incident wave. In the mesosphere, summer and winter echoes occur [Cho, Kelley, 1993; Kirkwood, 2007] and meteors regularly burn out leaving behind plasma trails existing for a few seconds. The main principle of MST radars is based on scattering by turbulent fluctuations with scales equal to half-wavelength. From the Gaussian waveform we determine intensity, Doppler shift, and spectrum width. This allows us to measure the turbulent energy dissipation rate, full neutral wind velocity vector, and IGW characteristics of specular atmospheric layers and mesospheric echo [Hocking, 2011]. IS radars generally have a sufficient energy potential for MST studies, but there are a number of other requirements. Thus, the MST radar should have a radio horizon as short as pos- sible, i.e. the maximum distance at which emitted electromagnetic waves are expected to be reflected from terrain objects. Furthermore, tropospheric and stratospheric research requires a high pulse repetition frequency. To effectively study the mesosphere and lower thermosphere, we should form alternating sequences to avoid range ambiguity when the height increases and maintain the required time resolution (see, e.g., [Farley, 1972]).

Recently, more and more artificial satellites and, consequently, space debris of various sizes have appeared in near-Earth space. Besides atmospheric research, the IS-MST radar will be used for systematic monitoring of space objects (SO). The phased array of the radar allows us to electronically control the beam, immediately switching over to another direction of transmission and reception. This makes it possible to monitor SO directly in the course of atmospheric and ionospheric observations. The radar is expected to monitor cataloged objects and search for unknown objects.

The antenna array of the radar allows us to conduct radio astronomical observations in a passive mode. Occurrence of ionospheric irregularities affects the signal from powerful cosmic radio sources, thus producing ionospheric scintillation [Yeh, Liu, 1982]. The field of view of the IS-MST radar covers such sources as Cygnus-A, Cassiopeia-A, and Crab Nebula, in which the scintillation effect is pronounced. Flexibility of operating modes of the IS-MST radar enables us to conduct simultaneously active and passive experiments, alternating periods of transmission and passive reception. Also of interest are solar observations. Solar activity may lead to the occurrence of radio bursts sudden changes of solar flux. The bursts accompany various physical processes on the Sun: coronal mass ejections, flares, and appearance of active regions. The intensity of the bursts may be several orders of magnitude higher than the radiation of the quiet Sun. Detection of such events with the IS-MST radar is particularly interesting because in VHF there are few solar radiometers with a large antenna area.

The IS-MST radar is unique also in terms of location as in Russia there is no instrument capable of making measurements throughout the atmosphere. There are about ten IS radars in the world, most of them located in the polar regions. There are new instruments - for example, an IS radar has recently been built in China [Ding et al., 2018]. EISCAT-3D radar complex is under construction, which will provide three-dimensional images of the state of ionospheric plasma [McCrea et al., 2015]. There are many troposphere-stratosphere radars, but few fully-featured MST radars because requirements for transmitter power and effective antenna area in mesospheric measurements increase significantly. Even a smaller number of instruments can make IS and MST measurements at a time. Similar measurements were made with the MU radar in Japan. A new MST radar MAARSY, located in Norway, can perform measurements of both the types [Latteck el al., 2012]. The PANSY radar was originally designed as an IS/MST radar for atmospheric research over Antarctica [Sato et al., 2014]. 
Also noteworthy is the interwork between the ISMST radar and other instruments of NHC RAS. Under the project, we plan to build a heater. This instrument will expand the research potential of the radiophysical observatories exploring the upper atmosphere. A powerful excitation wave of the heater gives rise to various phenomena, disturbances, and irregularities in the ionosphere [Streltsov et al., 2018]. The IS radar is the main diagnostic instrument to study artificial influence on the ionosphere. As part of NHC RAS at the radar site, we plan to deploy a lidar covering stratospheric and mesospheric heights. It will provide information on the neutral component of the atmosphere: chemical composition, neutral wind velocity, temperature, etc. Lidar data will be supplemented with IS-MST radar measurements, providing a more complete picture of atmospheric processes.

Next, we describe the IS-MST radar project, consider adopted technical solutions and operating modes, and evaluate its diagnostic potential for different applications.

\section{FREQUENCY RANGE OF THE RADAR}

Modern MST and IS radars cover a frequency range $45-1300 \mathrm{MHz}$. It is impossible to cover such a wide frequency range, ensuring in this case the required technical characteristics of the radar such as transmitted power, matching of antenna array elements, and requirements to the field of view. To achieve the objectives, we should carefully select the optimal operating frequency.

Most MST radars operate at frequencies near $50 \mathrm{MHz}$ [Hocking, 1997]. This is due to the power of backscatter by turbulent fluctuations in the neutral troposphere/stratosphere and in the ionized mesosphere. The turbulent mixing in the troposphere, stratosphere, and mesosphere gives rise to a refractive index gradient. During propagation of an electromagnetic wave with a length equal to twice the typical scale of the refractive index fluctuations, the Bragg scattering occurs. The scattering intensity depends on the frequency distribution of the energy of turbulent fluctuations. The fluctuation spectrum varies with height and is divided into three intervals. In the first long-wavelength range, the air kinetic energy is converted into turbulent fluctuations. In the inertial range, the energy gradually decreases with scales of eddies according to the law $k^{-5 / 3}$. The last portion of the spectrum is the viscosity range, where there is a sharp decay $\left(k^{-7}\right)$ of turbulences and energy is converted into heat. The maximum spectrum energy also depends on height, and above 25-30 km it decreases, thus making observations above this boundary unreasonable. As a result, the main region, where we can perform measurements, is a height range 5-25 km, the maximum scattering observed at $\sim 50 \mathrm{MHz}$ (fluctuations with scales of $\sim 3 \mathrm{~m}$ ). In the mesosphere, the turbulent scattering increases again due to increasing electron density, but the maximum energy of the fluctuations shifts toward larger characteristic scales $(10-1000$ m) [Hocking, 1985] and the selection of operating frequency becomes decisive. Furthermore, in summer and winter in the mesosphere, phenomena periodically occur which are called polar mesospheric echoes, observed even by UHF radars [Rapp et al., 2008]. The echo is sometimes accompanied by noctilucent clouds, which are visible to the naked eye. The clouds are generally formed at $3-4 \mathrm{~km}$ below the height of occurrence of the mesospheric echo. Initially the echo was observed only in polar regions but later also at middle latitudes. For example, in measurements carried out by Zeller et al. [2006], the echo occurred in $7 \%$ of the total observation time. The mesospheric echo is still poorly known, and its research requires complex optical and radiophysical instruments [Lübken et al., 2016].

Modern IS radars operate in VHF and UHF wavelengths [Robinson, 2004]. In the lower part of the range, the operating frequency is bounded by the ionospheric plasma frequency, at which the emitted wave is totally reflected (20-30 MHz), and by the sky noise level. If at $300 \mathrm{MHz}$ and $150 \mathrm{MHz}$ the cosmic radio noise temperature is respectively $100 \mathrm{~K}$ and $300 \mathrm{~K}$, at $50 \mathrm{MHz}$ it is 10 $000 \mathrm{~K}$ and the signal/noise ratio is reduced significantly. Given the weak IS signal and the need for measurements at $1000 \mathrm{~km}$, the sky noise becomes a major limiting factor. The upper boundary of the operating frequency range is determined by the condition of applicability of the IS method: the wavelength should be much larger than the Debye radius. In ionospheric plasma, the Debye radius increases with height, and by $1000 \mathrm{~km}$ it may be as large as $10 \mathrm{~cm}$. For studies with the IS method, the optimum operating frequencies are therefore in the range $200-500 \mathrm{MHz}$.

Thus, it is impossible to select the operating frequency appropriate both for MST measurements and for the IS method. A compromise solution is the range of $\sim 150 \mathrm{MHz}$. ISTP SB RAS possesses the Irkutsk IS radar (IISR), which is located $240 \mathrm{~km}$ east of the IS-MST radar and operates in a frequency range 152-164 MHz [Medvedev, Potekhin, 2019]. Due to the fact that in the Irkutsk region there are practically no sources of radio emission operating in the range $150-170 \mathrm{MHz}$, there is no need for additional clearing of radio spectrum at a new frequency. In addition, ISTP SB RAS has many devices, electronic components, and signal processing techniques applicable to the range of $\sim 150 \mathrm{MHz}$, e.g. the unique method of determining the electron density from the rate of electromagnetic wave polarization plane rotation (Faraday effect) [Shpynev, 2004]. It allows us to determine the electron density without calibration, and it is efficient in the frequency band from 120 to $170 \mathrm{MHz}$ since it is in this band the Faraday effect is pronounced in the received signal, which is necessary for solving the inverse problem.

\section{TECHNICAL DESCRIPTION OF THE RADAR}

At present, we have a preliminary design of the radar and are preparing for its construction. The radar will be located near Lake Baikal in Tazheran steppes (Kharikta area). The choice of the location is due firstly to the natural cavity in order to reduce the radio horizon of the radar. From the distance to the nearest hills and mountains on a topographic map we have calculated that the IS-MST radio horizon will be not larger than $10 \mathrm{~km}$. 
Secondly, measurements of electromagnetic compatibility in the frequency band of interest have shown that in this area the noise level is minimum. In addition, we can observe the state of the troposphere and stratosphere directly over the lake. This will help us to develop climate models.

Figure 1 shows the general view of the IS-MST radar. Two phased arrays are spaced by $100 \mathrm{~m}$ and have a tilt of $20^{\circ}$ in opposite directions so as to cover as large area as possible. Nevertheless, a change in efficiency of each array in the vertical direction is small. Between the antenna arrays are containers with transceiver equipment, elements of control, synchronization, and recording of received signals. In the middle is the control and data collection center, which will receive telemetry from the entire radar system, specify the waveform of emitted signals for the current operating mode, and primarily process received quadratures.

Table 1 lists the main characteristics of the IS-MST radar. Each antenna array of the radar includes 1536 cross dipoles, located at nodes of triangular lattice. When working simultaneously with two arrays in one direction, we can achieve a peak transmitter power of $2 \mathrm{MW}$ in a narrow beam of size $2.5^{\circ} \times 2.5^{\circ}$. Phased arrays can control the beam electronically, so we can get parameters in several vertical slices of the atmosphere, alternately transmitting and receiving signals in several directions. The cross dipoles allow us to separately receive two orthogonal polarizations. The excitation signal is fed to only one of the antenna dipoles to form a linearly polarized wave. This is necessary for the application of the method of determining the absolute electron density [Shpynev, 2004].

The two antenna arrays, located at a distance of $100 \mathrm{~m}$, form an interferometric base required to study the fine structure of processes in the neutral atmosphere and ionosphere, obtain accurate orbital SO characteristics, and improve angular resolution for radioastronomical observations. The radar baseline extends along magnetic field lines; one of the antenna arrays is northward; the other, southward. The field of view of each antenna array is $\pm 45^{\circ}$ from the main direction.

Due to the tilt of the arrays, the north-south field of view expands to $\pm 65^{\circ}$. All this will allow a detailed study of the processes that occur along magnetic field lines.

The transmitted signal waveform is determined by the operating mode and is formed in a digital synthesizer, after which the signal is converted to analog form and is split into transmitters.

Analog phase shifting is performed there in accordance with the desired direction, and after transmission a $2.5^{\circ} \times 2.5^{\circ}$ beam is formed. The radar has a general synchronization system in which different clock signals are split and arrive at separate transceiver modules.

The digital receiver system is more complex each phased antenna array is divided into 12 subarrays of 128 antennas each. The subarrays are connected to separate independent transceiver modules, where a partial beamforming occurs. This yields one receiving channel. Thus, at the output of one antenna array there are 12 receiving channels available. A subarray comprises only

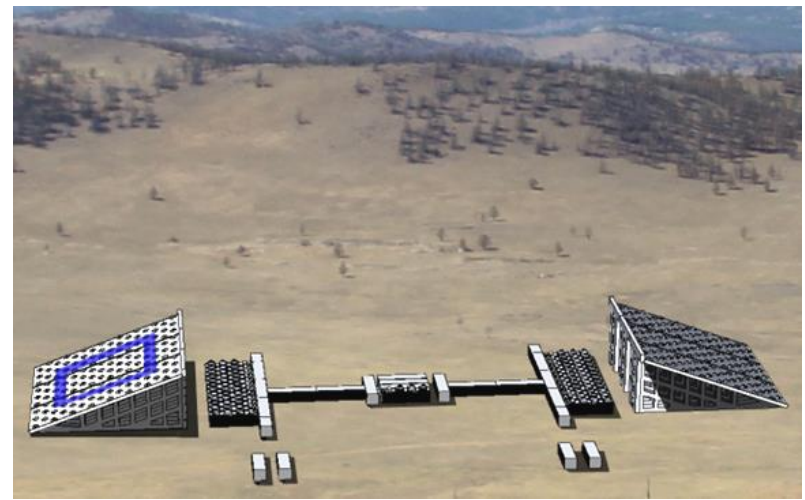

Figure 1. General view of the IS-MST radar

Table 1

Main technical characteristics of the IS-MST radar

\begin{tabular}{|l|c|}
\hline Operating frequency band & $154-162 \mathrm{MHz}$ \\
\hline Antenna array size & $40 \times 40 \mathrm{~m}$ \\
\hline $\begin{array}{l}\text { Number of elements } \\
\text { of antenna array }\end{array}$ & 1536 \\
\hline Type of antenna element & Cross dipole \\
\hline Peak transmitted power of one array & $1 \mathrm{MW}$ \\
\hline Number of arrays & 2 \\
\hline Antenna array effective area & $1250 \mathrm{~m}{ }^{2}$ \\
\hline System noise temperature & $\sim 500 \mathrm{~K}$ \\
\hline Beamwidth of one array & $2.5^{\circ} \times 2.5^{\circ}$ \\
\hline Receiver bandwidth & $625 \mathrm{kHz}$ \\
\hline Minimum height resolution & $480 \mathrm{~m}$ \\
\hline Antenna array field of view & $\pm 45^{\circ}$ \\
\hline
\end{tabular}

128 adjacent antennas, so the beam for such a channel is 12 times wider than the main lobe. Later, during preprocessing we can sum receiving channels with different phases, thus obtaining narrow receiving beams $2.5^{\circ} \times 2.5^{\circ}$ in different directions. This feature can be used for improving the angular resolution at the reception, for simultaneous operation in active and passive modes, as well as for examining a wide area irradiated by the heater, which has a wider main lobe. Moreover, the receiving phased array system is fully digital. Signals from individual antennas are amplified in a low noise amplifier, are filtered, and digitized. By reducing the number of analog components, we can avoid distortion of received signal. In turn, the modern element base provides a large dynamic range required to measure both powerful echo signals at low heights and weak IS signals from plasmaspheric heights.

\section{OPERATING MODES OF THE RADAR}

The radar with large antenna area and high radiated power is a rare instrument, which, as shown above, can be used to solve many scientific and applied problems. To get the most out of the radar, we must competently 
approach the selection of operating modes. Figure 2 schematically shows the main operating modes: atmospheric research, SO monitoring, cooperative observations, and passive radioastronomical observations. Table 2 lists settings of operating modes for regular studies of the lower and middle atmosphere (MST) and ionosphere (IS-1, IS-2). It also shows such characteristic as the product of mean radiated power and effective area $P A$, which is often employed to evaluate the diagnostic potential of radars.

When the radar operates in the MST mode (Figure 2, a), the basic observed atmospheric parameter is the neutral wind velocity. To obtain a full velocity vector, the radar must operate in three directions at a time. Since the troposphere/stratosphere are at a short distance from the radar, the pulse repetition frequency may be high. The turbulent scattering signal has a narrow spectrum of the order of several hertz, so statistical characteristics of the signal vary slightly. Parameters of atmospheric scattering are usually estimated by calculating the autocorrelation function $(\mathrm{ACF})$ of received signal and by de-
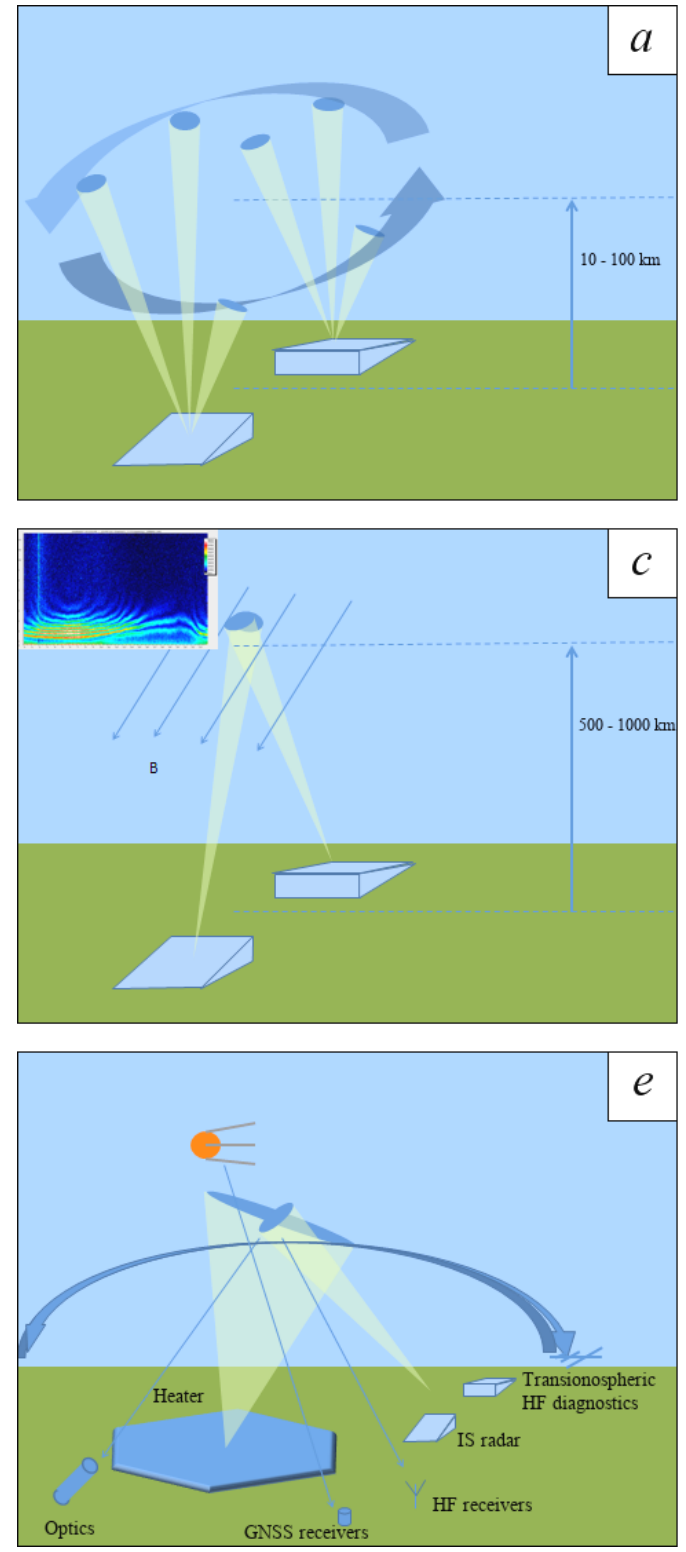

Figure 2. Operating modes of IS-MST: MST mode (a); IS-1 mode $(b)$; IS-2 mode $(c)$, SO monitoring mode $(d)$; cooperative observation mode $(e)$, radioastronomical mode $(f)$

termining the radial velocity from its phase. The velocity determination variance is, however, high, hence the need for signal accumulation [Zrnic, 1979]. Signals from individual pulses are coherently summed in an interval of time, then ACF and atmospheric parameters are evaluated. In the troposphere and stratosphere, there is no need for high $P A$, and measurements can be made using one antenna array or even its part. In the mesosphere, a mode with large $P A$ is required.

For ionospheric research, we will utilize two operating modes: IS-1 (Figure 2, $b$ ) and IS-2 (Figure 2, c) corresponding to two height ranges: below $500 \mathrm{~km}$ and above $500 \mathrm{~km}$. This division follows from the experience of IS measurements with IISR. The scattering intensity at heights up to $500 \mathrm{~km}$ is large enough for stable estimation of ionospheric plasma parameters; we can, therefore, increase the time resolution by averaging a received signal to a lesser degree. In the IS mode, signal coherence is less than the time for pulse propagation, hence we cannot apply the coherent accumulation.
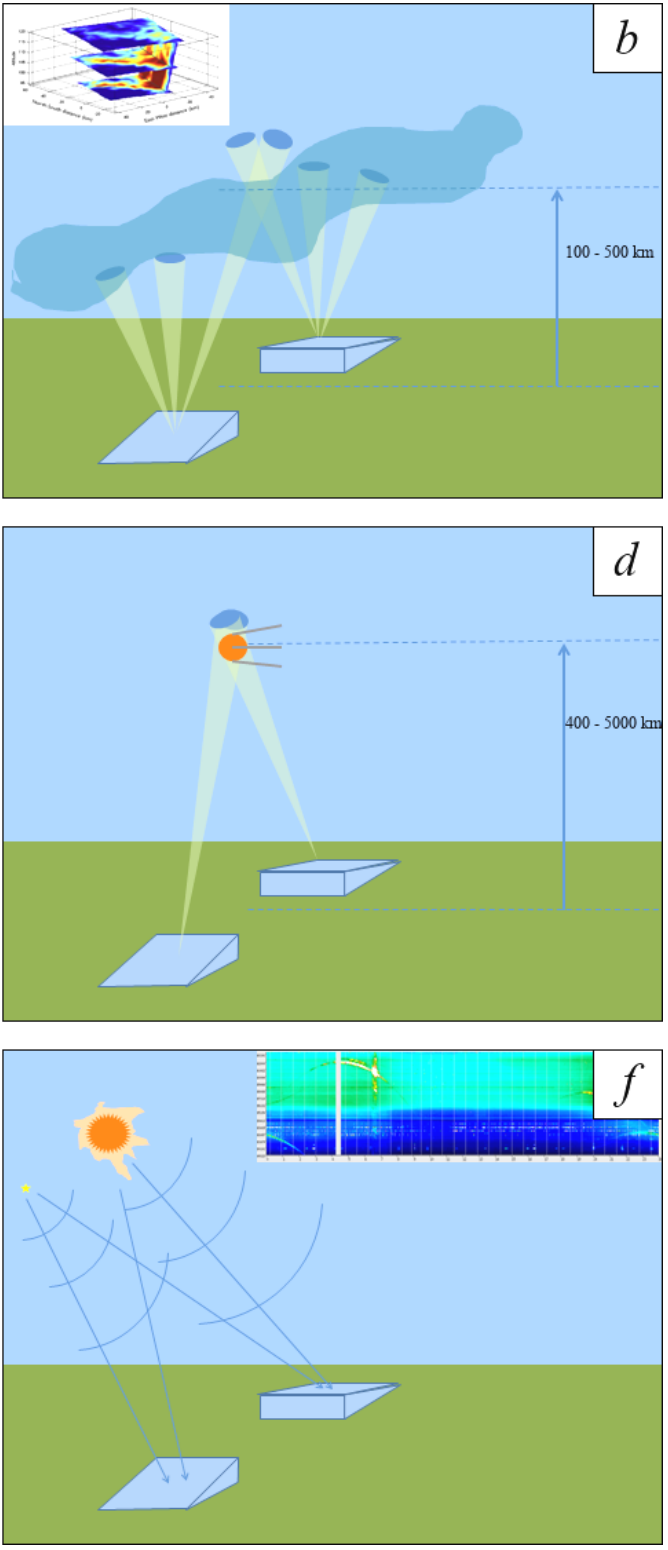
Table 2

Modes of IS-MST operation for atmospheric research

\begin{tabular}{|l|c|c|c|}
\hline & $\begin{array}{c}\text { MST } \\
\text { mode }\end{array}$ & $\begin{array}{c}\text { IS-1 } \\
\text { mode }\end{array}$ & $\begin{array}{c}\text { IS-2 } \\
\text { mode }\end{array}$ \\
\hline $\begin{array}{l}\text { Pulse repetition } \\
\text { frequency, Hz }\end{array}$ & 1333.3 & 250 & 75 \\
\hline Pulse duration, $\mu$ s & 51.2 & 700 & 6500 \\
\hline Duty cycle, \% & 6.8 & 17.5 & 37.5 \\
\hline $\begin{array}{l}\text { Product of mean power } \\
\text { and effective area, } \\
\mathrm{W} \cdot \mathrm{m}^{2}\end{array}$ & $3.4 \cdot 10^{8}$ & $8.75 \cdot 10^{8}$ & $18.75 \cdot 10^{8}$ \\
\hline
\end{tabular}

Scattering ACF is estimated from one pulse, and then ACFs from different pulses are averaged. It is, therefore, required that the pulse length be large. To maintain the high height resolution, complex signals are applied. The plasma drift velocity in the IS-1 mode is estimated using three beams as in the MST mode. At heights from $500 \mathrm{~km}$, an IS signal is significantly attenuated, and to maximize the energy potential of the radar we use a large pulse length, a high duty cycle, and two phased arrays in one direction.

In the SO monitoring mode (see Figure $2, d$ ), one or both beams of the radar monitor predetermined objects with known orbital characteristics or operate in the search mode to detect unknown SO. During regular IS measurements, we will also continuously monitor the appearance of SO in the field of view of the ISMST radar. Figure 2, $e$ shows the cooperative observation mode when an ionospheric region, irradiated by the heater, is scanned by the IS-MST radar, optical means, HF transceivers, and GNSS-receiver system. The location for the NHC instruments was selected to carry out similar experiments on monitoring the state of the atmosphere. The last of the mode shown in Figure $2, f$ is radioastronomical observations when the radar does not transmit but detects space signals from different directions. In this mode, we can take full advantage of the beamforming capability of the radar by using several narrow receiving beams simultaneously, and can utilize interferometric capabilities. In addition to these modes, we will conduct special experiments, for example, on monitoring the coherent echo.

For two decades of dealing with IISR data, many methods of IS-signal processing, SO monitoring, and passive observations have been developed. Shpynev [2004] has described a method of determining the electron density from the angle of polarization plane rotation. Berngardt, Kushnarev [2013] offer a method of increasing the height resolution in estimating ionospheric plasma parameters, which is based on the difference between pulses of different duration. Shcherbakov et al. [2009] show the method of determining plasma drift velocity, which is applied in IISR. Medvedev et al. [2013] combine IISR and digisonde data in order to identify characteristics and direction of propagation of traveling ionospheric disturbances. IISR capabilities and methods used for monitoring space debris are described in [Khakhinov et al., 2009]. To filter clutter signals, a method has been worked out which is based on coherent summation of received signal [Tashlykov et al., 2019]. A method of estimating the solar flux, which relies on calibration using powerful radio sources and sky noise maps, is presented in [Setov et al., 2018]. Vasilyev et al. [2017] from scintillation of the radio source Cygnus-A have determined the scintillation index S4 and have estimated the zonal velocity of ionospheric irregularities at the height of maximum electron density in the F2 layer.

\section{ASSESSING THE POTENTIAL OF THE RADAR}

We have discussed the selection of frequency band for the IS-MST radar and have shown that there is no operating frequency appropriate for all radar applications. A compromise solution is a frequency band of $\sim 150 \mathrm{MHz}$. Nevertheless, it is important to evaluate capabilities of the radar for operating at this frequency in the main operating modes.

In assessing the potential for the MST, IS-1, and IS2 modes, we can start from volume reflectivity. This characteristic depends only on atmospheric parameters, thereby providing a vertical profile of the volume reflectivity in the atmosphere. On the other hand, from specifications of the radar we can find the minimum volume reflectivity, which can be used to determine an atmospheric parameter. Thus, we can find a height range within which we can make the measurements. The criterion for estimating atmospheric parameters is the desired statistical accuracy of results. The key characteristic in estimating the potential is, therefore, the signal/noise ratio $(S N R)$. SNR required to ensure the desired statistical accuracy can be found analytically or through modeling. Next, we present estimates of the potential for atmospheric modes of operation. The method of estimating the potential and the findings are described in more detail in [Potekhin et al., 2016]. The atmosphere was simulated using parameters of the NRLMSIS-00 and IRI-2012 models.

Turbulent scattering includes a large number of atmospheric parameters. In the mesosphere, it increases due to additional ionized component [Lübken, 2014]. The main parameter of interest is the neutral wind velocity, therefore $S N R$ was set so that the standard deviation of the obtained velocity in the troposphere and stratosphere did not exceed $1 \mathrm{~m} / \mathrm{s}$; in the mesosphere, $2 \mathrm{~m} / \mathrm{s}$. With given simulation parameters, the IS-MST radar will allow us to measure the neutral wind velocity in the troposphere and stratosphere at heights up to $25 \mathrm{~km}$. In the mesosphere in summer, we can make measurements at $\sim 86 \mathrm{~km}$ for increasing Schmidt number, which characterizes the degree of electron diffusion attenuation due to the occurrence of ionized ice particles. This is believed to be one of the causes of the summer mesospheric echo. We also demonstrate that it is possible to observe the winter mesospheric echo, which occurs at lower heights $(\sim 70 \mathrm{~km})$ and does not require an increased Schmidt number. It is in the mesosphere where the advantage of the $50 \mathrm{MHz}$ frequency, adopted in most MST radars, is evident - scattering in this case is stronger and covers a much larger height range. 
In IS measurements, the emphasis is on the determination of electron density, temperature, and plasma drift velocity. We believe assume that the relative standard deviation in the determination of density and temperatures should be within $10 \%$, and the standard deviation of the plasma drift velocity - the parameter known for its high variance - should not exceed $45 \mathrm{~m} / \mathrm{s}$. The simulation has shown that these parameters can be measured in the IS-1 mode at all operating heights $100-500 \mathrm{~km}$. In the IS-2 mode, the electron density can be measured at heights slightly higher than $1500 \mathrm{~km}$; the temperature, at heights to $1320 \mathrm{~km}$; the plasma drift velocity, to $1140 \mathrm{~km}$.

Sensitivity of the IS-MST radar in the mode of interferometric measurements can be estimated from the maximum derivative of the correlation coefficient when the slope of the phase-angle response of the interferometer is less steep. We have shown that the antenna system of the IS-MST radar is most sensitive to objects with an angular size of $7.3 \mathrm{arcmin}$, which is sufficient for the study of the fine structure of atmospheric irregularities.

A wide field of view, high pulse repetition frequency, large antenna area, and high radiated power make the IS-MST radar a good instrument for monitoring SO. In the experience of detection of SO with IISR, the ISMST radar is appropriate for measuring orbit parameters with the accuracy required for the forecast of the next revolution of an object. At present, for the measurement of SO coordinate information with IISR the standard deviation in range is $\sim 100-300 \mathrm{~m}$, the standard deviation in target angles is $\sim 2-5$ arcmin. The IS-MST radar has a more advanced configuration, so the accuracy of measurements can be expected to be higher.

Radioastronomical sensitivity of one antenna array when averaging a received signal for $0.1 \mathrm{~s}$ is $4.4 \cdot 10^{-26}$ $\mathrm{W} /\left(\mathrm{m}^{2} \cdot \mathrm{Hz}\right)$. To monitor radio sources, the IS-MST radar has a low angular resolution and only one extended baseline, but due to the high sensitivity we can measure a flux from powerful radio sources. The IS-MST radar is unique for this problem due to its operating frequency of $158 \mathrm{MHz}$ - there are few instruments operating in VHF with large effective antenna area in the world.

\section{CONCLUSION}

At present, for studies of processes and phenomena occurring in the atmosphere and near-Earth space it is not enough to have instruments operating only in a local region or in a small height range. To obtain a full picture, instruments are required which can cover different layers, frequency bands, and time spans. That is why NHC RAS includes several modern multi-purpose facilities for studying solar-terrestrial relations. Among them, the IS-MST radar can cover the maximum height range in the atmosphere and receive the parameters that cannot be determined by other instruments. The radar will allow us to solve topical fundamental problems of solar-terrestrial physics, to study dynamics and wave activity in the lower and middle atmosphere, magnetosphere-ionosphere relations, and energy transfer processes in the ionosphere-mesosphere-stratosphere- troposphere system. Furthermore, it will regularly monitor SO and solar activity in VHF. The all-atmosphere IS-MST radar will fill a gap in the network of IS and MST radars, covering south-eastern Siberia.

This study was supported by the Grant of the Russian Science Foundation (Project No. 18-17-00042). The observational results were obtained using the equipment of Center for Common Use «Angara» (http://ckp-rf.ru/ckp/3056/) within budgetary funding of Basic Research program II.12.

The solar flux data measured at Learmonth observatory is available at ftp-server of Australian Government Bureau of Meteorology ftp://ftp-out.sws.bom.gov.au.

\section{REFERENCES}

Berngardt O.I., Kushnarev D.S. Effective subtraction technique at the Irkutsk Incoherent Scatter Radar: Theory and experiment. J. Atmos. Solar-Terr. Phys. 2013, vol. 105-106, pp. 293-298. DOI: 10.1016/j.jastp.2013.03.023.

Cho J.Y.N., Kelley M.C. Polar mesosphere summer radar echoes: observations and current theories. Rev. Geophys. 1993 , vol. 31, no. 3, pp. 243-265. DOI: 10.1029/93RG01535.

Ding Z., Wu J., Xu Z., Xu B., Dai. L. The Qujing incoherent scatter radar: system description and preliminary measurements. Earth, Planets and Space. 2018, vol. 70, iss. 1, article id. 87, 13 p. DOI: 10.1186/s40623-018-0859-8.

Evans J.V. Theory and practice of ionosphere study by Thomson Scatter Radar. Proc. IEEE. 1969, vol. 59, no. 4, pp. 496-530. DOI: 10.1109/PROC.1969.7005.

Farley D.T. Multiple-pulse incoherent-scatter correlation function measurements. Radio Sci. 1972, vol. 7, no. 6, pp. 661-666. DOI: 10.1029/RS007i006p00661.

Hocking W.K. Measurement of turbulent energy dissipation rates in the middle atmosphere by radar techniques: A review. Radio Sci. 1985, vol. 20, no. 6, pp. 1403-1422. DOI: 10.1029/RS020i006p01403.

Hocking W.K. Recent advances in radar instrumentation and techniques for studies of the mesosphere, stratosphere and troposphere. Radio Sci. 1997, vol. 32, no. 6, pp. 2241-2270. DOI: 10.1029/97RS02781.

Hocking W.K. A review of mesosphere-stratospheretroposphere (MST) radar developments and studies, circa 19972008. J. Atmos. Solar-Terr. Phys. 2011, vol. 73, pp. 848-882. DOI: 10.1016/j.jastp.2010.12.009.

Khakhinov V.V., Lebedev V.P., Medvedev A.V., Ratovsky K.G. Capabilities of the Irkutsk Incoherent Scattering Radar for space debris studies. Proc. $5^{\text {th }}$ European Conference on Space Debris. Darmstadt, Germany. 2009, vol. 5, no. 1, 7 p.

Kirkwood S. Polar mesosphere winter echoes - A review of recent results. Adv. Space Res. 2007, vol. 40, pp. 751-757. DOI: 10.1016/j.asr.2007.01.024

Latteck R., Singer W., Rapp M., Vandepeer B., Renkwitz T., Zecha M., Stober G. MAARSY: The new MST radar on Andøya - System description and first results. Radio Sci. 2012, vol. 47, RS1006. DOI: 10.1029/2011RS004775.

Lübken F.-J. Turbulent scattering for radars: A summary. J. Atmos. Solar-Terr. Phys. 2014, vol. 107, pp. 1-7. DOI: 10.1016/j.jastp.2013.10.015.

Lübken F.-J., Latteck Ralph, Becker E., Höffner J., Murphy D. Using polar mesosphere summer echoes and stratospheric/mesospheric winds to explain summer mesopause jumps in Antarctica. J. Atmos. Solar-Terr. Phys. 2016, vol. 162, pp. 106-115. DOI: 10.1016/j.jastp.2016.06.008.

McCrea I., Aikio A., Alfonsi L., Belova E., Buchert S., Clilverd M., et al. The science case for the EISCAT_3D radar. Progress in Earth and Planetary Science. 2015, vol. 2. DOI: 10.1186/s40645-015-0051-8. 
Medvedev A.V., Potekhin A.P. Irkutsk Incoherent Scatter Radar: history, present and future. Hist. Geo Space Sci. 2019, vol. 10. DOI: 10.5194/hgss-10-215-2019.

Medvedev A.V., Ratovsky K.G., Tolstikov M.V., Alsatkin S.S., Scherbakov A.A. Studying of the spatialtemporal structure of wavelike ionospheric disturbances on the base of Irkutsk Incoherent Scatter Radar and digisonde data. J. Atmos. Solar-Terr. Phys. 2013, vol. 105-106, pp. 350-357. DOI: 10.1016/j.jastp.2013.09.001.

Potekhin A.P, Setov A.G., Lebedev V.P., Medvedev A.V., Kushnarev D.S. Prospective IS-MST radar. Potential and diagnostic capabilities. J. Solar-Terr. Phys. 2016, vol. 2, no. 3, pp. 3-21. DOI: $10.12737 / 22281$.

Rapp M., Strelnikova I., Latteck R., Hoffmann P., Hoppe U.-P., Häggström I., Rietveld M.T. Polar mesosphere summer echoes (PMSE) studied at Bragg wavelength of $2.8 \mathrm{~m}, 67 \mathrm{~cm}$, and $16 \mathrm{~cm}$. J. Atmos. Solar-Terr. Phys. 2008, vol. 70, pp. 947-961. DOI: 10.1016/j.jastp.2007.11.005.

Robinson R. New Techniques and Results from Incoherent Scatter Radars. URSI Radio Sci. Bull. 2004, vol. 2004, iss. 311, pp. 79-94. DOI: 10.23919/URSIRSB.2004.7909637.

Sato K., Tsutsumi M., Sato T., Nakamura T., Saito A., Tomikawa Y., et al. Program of the Antarctic Syowa MST/IS radar (PANSY). J. Atmos. Solar-Terr. Phys. 2014, vol. 118, pp. 2-15. DOI: 10.1016/j.jastp.2013.08.022.

Setov A.G., Globa M.V., Medvedev A.V., Vasilyev R.V., Kushnarev D.S. First results of absolute measurements of solar flux at the Irkutsk Incoherent Scatter Radar (IISR). Solar-Terr. Phys. 2018, vol. 4, no. 3, pp. 24-27. DOI: $10.12737 / \mathrm{stp}-43201804$.

Shcherbakov A.A., Medvedev A.V., Kushnarev D.S. Correlation method for determining the ionospheric plasma drift velocity at the Irkutsk Incoherent Scatter Radar. Geomag netism and Aeronomy. 2009, vol. 49, no. 7, pp. 1028-1033. DOI: $10.1134 /$ S0016793209070317.

Shpynev B.G. Incoherent scatter Faraday rotation measurements on a radar with single linear polarization. Radio Sci. 2004, vol. 39, RS3001. DOI: 10.1029/2001RS002523.
Streltsov A.V., Berthelier J.-J., Chernyshov A.A., Frolov V.L., Honary F., Kosch M.J., et al. Past, present and future of active radio frequency experiments in space. Space Sci Rev. 2018, vol. 214, no. 118. DOI: 10.1007/s11214-018-0549-7.

Tashlykov V., Setov A., Medvedev A., Lebedev V., Kushnarev D. Ground clutter deducting technique for Irkutsk Incoherent Scatter Radar. Proc. 2019 Russian Open Conference on Radio Wave Propagation (RWP). 2019, pp. 175-178. DOI: 10.1109/RWP.2019.8810369.

Vasilyev R., Globa M., Kushnarev D., Medvedev A., Ratovsky K. Spectral characteristics of ionospheric scintillations of VHF radiosignal near magnetic zenith. J. Atmos. Solar-Terr. Phys. 2017, vol. 160, pp. 48-55. DOI: 10.1016/j.jastp.2017.05.016.

Yeh K.C., Liu C.-H. Radio wave scintillations in the ionosphere. Proc. the IEEE. 1982, vol. 70, no. 4, pp. 324-360. DOI: 10.1109/PROC.1982.12313.

Zeller O., Zecha M., Bremer J., Latteck R., Singer W. Mean characteristics of mesosphere winter echoes at mid- and high latitudes. J. Atm. and Solar-Terr. Phys. 2006, vol. 68, pp. 1087-1104. DOI: 10.1016/j.jastp.2006.02.015.

Zrnic D. Estimation of spectral moments for weather echoes. IEEE Trans. Geosci. Electronics. 1979, vol. GE-17, no. 4, pp. 113-128. DOI: 10.1109/TGE.1979.294638.

How to cite this article

Medvedev A.V., Potekhin A.P., Setov A.G., Kushnarev D.S., Lebedev V.P. All-atmosphere IS-MST radar. Solar-Terrestrial Physics. 2020. Vol. 6. Iss. 2. P. 41-48. DOI: $10.12737 /$ stp-62202004. 